\title{
Wahrhaftigkeit als Definition der Kirche im Matthäusevangelium
}

François Vouga

\section{Versuch einer Definition: Wahrhaftigkeit als Ge- rechtigkeit}

Die Suche nach einem Äquivalent für den Begriff der »Wahrhaftigkeit " in der griechischen Sprache und in der neutestamentlichen Theologie erweist sich als eine hermeneutische Herausforderung. Wahrscheinlich könnten wir nach wesentlichen Dimensionen der johanneischen Definition der Liebe oder nach den Implikationen der Wahrheit in den Paulusbriefen fragen. Ein besonders anregender Weg scheint mir jedoch die matthäische Reflexion über die Kommunikationsethik, die uns vielleicht nicht ganz ohne Provokation zu der matthäischen Definition des besonderen Raumes der ekklesia, der Kirche führt, zu eröffnen.

Zugestanden: Dieser anregende Weg ist insofern aufregend, als er zunächst einen Umweg der Interpretation verlangt. Denn auch im Matthäusevangelium suchen die Leserin und der Leser vergeblich nach einem unmittelbaren Äquivalent. Naheliegend wäre es gewesen, in diesem Zusammenhang die matthäische Kritik der Heuchelei, der Hypokrisie, zu untersuchen. Eine solche Untersuchung führt aber deswegen an der Problematik der Wahrhaftigkeit vorbei, weil es den matthäischen Heuchlern an Wahrhaftigkeit nicht fehlt. Zu den Besonderheiten der Sprache des ersten Evangeliums gehört, dass es den Begriff systematisch benutzt, um objektive - und nicht subjektive Heuchler, zu warnen. ${ }^{1}$ Auffällig ist zunächst, dass Matthäus nicht das Abstraktum Heuchelei bzw. Hypokrisie verwendet²: Für ihn besteht nicht die Gefahr in einer Eigenschaft, sondern in der singulären Haltung konkreter Menschen, unter denen sich der Leser universal

1 Darauf macht in seinem klassisch gewordenen Kommentar sehr präzise aufmerksam Pierre Bonnard, L'évangile selon saint Matthieu, CNT I, Neuchâtel 1963, 79, 97, 322, 338.

2 Das Abstraktum Hypokrisie (Heuchelei) kommt nur je einmal in den synoptischen Evangelien vor: Mk 12,15; Mt 23,23 und Lk 12,1; weitere dreimal findet es sich im NT nur in Gal 2,13; 1 Tim 4,2 und 1 Petr 2,1. 
wieder erkennen könnte. ${ }^{3}$ Entscheidend ist dabei, dass für Matthäus die Menschen nicht Heuchler sind, weil sie Andere täuschen wollen, sondern weil sie sich in Wirklichkeit selbst täuschen und dass sie als Kollateralschaden Andere in aller Ehrlichkeit mit sich selbst in die Irre führen. Sie haben kein falsches Verhältnis zu der Wahrhaftigkeit, sondern zu der Wahrheit ihres Verhältnisses zu Gott und zu ihrer eigenen Situation.

Die matthäischen Heuchler sind Menschen, die wahrhaftig überzeugt sind, in der Gerechtigkeit zu leben, aber die Immanenz der Religion mit der Transzendenz der wundersamen Grosszügigkeit der Vorsehung Gottes verwechseln - aus einem ähnlichen Missverständnis wie die Lehrer in Galatien, die das Gesetz für Gott halten (Gal 3,21!). Um es noch präziser zu formulieren: Das Matthäusevangelium unterscheidet dadurch die vermeintliche Gerechtigkeit der Heuchler (Mt 6,1) von der Gerechtigkeit Gottes und kennzeichnet sie dadurch als eine unglückliche Parodie der Gerechtigkeit. Als blosse Demonstration "vor den Menschen" wird sie mit dem Ziel und mit der Konsequenz ausgeübt, dass sie ihren Lohn direkt von ihnen bekommt (Mt 6,1-18). Die Offenbarung des ewigen Unterschieds zwischen der Gerechtigkeit der Heuchler und der Gerechtigkeit Gottes sowie die Warnung der Lesenden vor der möglichen Verwechslung der Gerechtigkeit Gottes mit ihrer Parodie setzen hingegen ein matthäisches Verständnis der Gerechtigkeit voraus, das uns in die Nähe der Wahrhaftigkeit bringt. Der matthäische Begriff der Gerechtigkeit verweist nämlich auf ein sachgemässes Verhältnis zwischen den "Gerechten" und ihrem himmlischen Vater und, folglich, mit sich selbst und mit den Menschen. Die Gerechtigkeit besteht dann aus einem gegenseitigen Vertrauensverhältnis, in dem das menschliche Subjekt aus dem Vertrauen und durch das Vertrauen auf das Vertrauen gerecht wird, das der himmlische Vater in ihn oder in sie setzt. Diese Definition, die an die Definition erinnert, die Ferdinand Christian Baur vom paulinischen Begriff der Gerechtigkeit gegeben hatte, ${ }^{4}$ ermöglicht auf jeden Fall eine klare, logische - nicht widersprüchliche - und plausible Interpretation des matthäischen Sprachgebrauchs:

3 Mt 6,2.5.16; 7,5; 15,7; 22,18; 23,13.14.15.23.25.27.29; 24,51. Im NT nur noch Mk 7,6; Lk 6,42; 12,56;13,15.

4 Ferdinand Christian Baur,Vorlesungen über Neutestamentliche Theologie, Leipzig 1864, 133 .

5 Die Übersetzungen dieser Auflistung folgen Ulrich Luck, Das Evangelium nach Matthäus, ZBkNT 1, Zürich 1993. 
Mt 3,15: Jesus aber antwortete und sprach zu ihm [= Johannes]:

Lass es jetzt geschehen [= dass Jesus von Johannes getauft wird]; denn so kommt es uns zu, jede Gerechtigkeit zu erfüllen.

Mt 5,6: Selig sind die Hungernden und Dürstenden der Gerechtigkeit, denn sie werden gesättigt werden.

Mt 5,10: Selig sind die um der Gerechtigkeit willen Verfolgten, denn ihrer ist die Herrschaft der Himmel.

Mt 5,20: Wenn eure Gerechtigkeit nicht weit hinausgeht über die der Schriftgelehrten und Pharisäer, so werdet ihr nicht hineinkommen in die Herrschaft der Himmel.

Mt 6,1: Hütet euch,

eure Gerechtigkeit vor den Menschen zu tun, um von ihnen gesehen zu werden;

sonst habt ihr keinen Lohn bei eurem Vater in den Himmeln.

Mt 6,33: Sorgt euch nun nicht, indem ihr sprecht:

Was sollen wir essen?

oder: Was sollen wir trinken?

oder: Was sollen wir anziehen?

Denn nach alledem trachten die Heiden.

Denn euer himmlischer Vater weiss, dass ihr alles das braucht. Trachtet aber zuerst nach seiner Herrschaft und seiner Gerechtigkeit, und das alles wird euch dazugegeben werden.

Mt 21,32: Denn Johannes kam zu euch auf dem Wege der Gerechtigkeit, und ihr glaubtet ihm nicht.

Ich denke nicht, dass der matthäische Begriff der Gerechtigkeit und der deutsche Begriff der Wahrhaftigkeit gleichgesetzt werden können. Als hermeneutische Hypothese schlage ich aber vor, die Gerechtigkeit als die notwendige und ausreichende Bedingung zu lesen, die nach der matthäischen Interpretation des Evangeliums als frohe Botschaft ermöglicht, die Wahrhaftigkeit mit der Wahrheit zu verbinden. 


\section{Gerechtigkeit, Wahrhaftigkeit und Ethik der Kom- munikation: Mt 16,13-20,34}

Wenn die Gerechtigkeit das gegenseitige Vertrauensverhältnis bezeichnet, das Wahrhaftigkeit und Wahrheit miteinander verbindet, stellt sich die Frage, wie und wo sie als Wirklichkeit des Alltags gegeben und aufgebaut wird.

Naheliegend wäre es vielleicht gewesen, die matthäische Vorstellung der Gerechtigkeit mit einem Verweis auf den Begriff des Gesetzes näher zu bestimmen. Auch wenn man davon absieht, dass Matthäus sein Verständnis des Gesetzesbegriffes nie erklärt - mit guten Gründen liest ihn Jean Calvin mit der Bedeutung des Gotteswillens ${ }^{6}$, was ihn in die Nähe des paulinischen und markinischen Begriffes des Evangeliums rückt ${ }^{7}$-, muss man feststellen, dass die einzige Stelle, die beide in Verbindung setzt, genau umgekehrt argumentiert: Nicht das Gesetz definiert die Gerechtigkeit, sondern die Gerechtigkeit wird als das äussere Kriterium herangezogen, nach welchem die "Erfüllung des Gesetzes" gedacht werden soll (Mt 5,17-20). Die Gerechtigkeit impliziert, dass das Gesetz und die Propheten, das heisst die Schrift, vom Willen des Gesetzgebers her ausgelegt wird, kommentiert Calvin. ${ }^{8}$

Am besten hilft, glaube ich, die im ersten Evangelium klar, kritisch und präzise entwickelte Ethik der Kommunikation, die die Verheissung der Ekklesia definiert (Mt 16,13-20,34). Die Argumentation, die Matthäus frei auf der Basis der Vorlage des Markusevangeliums entfaltet, ist nach einer deutlichen eigenen Logik strukturiert. Vier Sequenzen können unterschieden werden:

1. Die erste Sequenz (Mt 16,13-17,22) legt den anthropologischtheologischen Grund der matthäischen Definition der Ekklesia. Es fällt auf, dass das erste Evangelium, das sich mit der Gründung der Kirche explizit befasst, sie weder institutionell noch dogmatisch definiert. Das Matthäusevangelium lässt vielmehr die Ekklesia in einem dialogischen Prozess entstehen, der die Voraussetzungen bildet für eine gegenseitige Anerkennung, für die Wahrnehmung einer neuen persönlichen Identität und der dazu gehörenden neuen Verhaltensweisen sowie für die Einladung zu einer Haltung des Vertrauens.

\footnotetext{
${ }^{6}$ Jean Calvin, Commentaires sur le Nouveau Testament I: Sur la concordance ou Harmonie composée de trois évangélistes asçavoir S. Matthieu, S. Marc et S. Luc, Paris 1854, 156.

7 Martin Stiewe/François Vouga, Die Bergpredigt und ihre Rezeption als kurze Darstellung des Christentums, NET 2, Tübingen 2001, 146-155.

8 Calvin, Commentaires, 157.
} 
2. Auf dieser Basis entwickelt die zweite Sequenz (Mt 17,2318,37) eine konsequente Ethik der Kommunikation, die die Ekklesia als Gemeinde des Vertrauens und der Wahrhaftigkeit aufbauen soll. Eine dialektische Definition der evangelischen Freiheit leitet drei pragmatische Gemeinderegeln ein:

- Die aktive Aufmerksamkeit auf die Kleinen und die Verantwortung, diejenigen, die sich verlieren, zurückzuholen;

- die als Gemeindedisziplin durchdachte Strategie einer konstruktiven, aufrichtigen und respektvollen Kritik;

- eine Radikalisierung des Vergebungsverständnisses, die von den Vollkommenheitsidealen befreit und die Möglichkeit eröffnet, mit der Freiheit und der Schwachheit der Anderen frei zu leben.

3. Die dritte Sequenz (Mt 19,1-20,16) inszeniert und löst Krisensituationen, die aus den Konflikten des Alltags in und an den Grenzen der Gemeinde entstehen:

- Mann und Frau;

- der Platz der Kinder;

- die Auseinandersetzung mit den eigenen Vollkommenheitsidealen;

- die zugrundeliegende Neudefinition der Gerechtigkeit.

4. In der vierten Sequenz (Mt 20,17-34) schliesst Matthäus seine ekklesiologische Reflexion mit einer kritischen Neudefinition der wahren Grösse in der Gemeinschaft der Ekklesia und mit der - symmetrisch zum ersten Dialog zwischen Jesus und Petrus - programmatischen Inszenierung einer Gemeinschaft von Blinden ab, die sich der Barmherzigkeit des Herren anvertrauen, die von ihm gerettet werden und ihm nachfolgen.

Ich möchte mich auf die beiden ersten Sequenzen konzentrieren, die sich mit der Definition der Kirche und ihrer Grundlage direkt befassen. 


\section{Erste Sequenz: Matthäus 16,13-17,20}

\section{Die gegenseitige Anerkennung des Dialogs als Gründung der Ekklesia}

1. Die Verheissung der Ekklesia als Raum der Gerechtigkeit wird auf dem Ereignis eines Dialogs gegründet, in dem ein gegenseitiges Vertrauen seinen Ausdruck in der Form einer gegenseitigen Namensgebung findet: Petrus bekennt Jesus als Christus, und Jesus schenkt Petrus das Vertrauen, ihn als den ersten Stein der neuen Gemeinschaft, die er aufbauen wird, zu benennen (Mt 16,15-18).

(15) Er sagte ihnen [seinen Jüngern]:

Ihr, wer, sagt ihr, bin ich?

(16) Simon Petrus antwortete:

Du bist der Christus, der Sohn des lebendigen Gottes!

(17) Jesus sagte ihm:

Glücklich bist du, Simon Barjona,

weil

Fleisch und Blut haben es dir nicht offenbart, sondern mein Vater, der in den Himmeln.

Und ich sage dir

dass du Petrus / Stein bist,

(18) und auf diesem Stein werde ich meine Kirche aufbauen.

Die literarische Komposition des vorliegenden Dialogs ist fiktional: In der Zeit der matthäischen Redaktion gehörten beide Gesprächspartner des Dialogs längst zurVergangenheit. Als prägende Figur, deren persönliche Geschichte und apostolische Wirkung abgeschlossen war und die am besten die universale Offenheit des frühen Christentums symbolisieren konnte, eignete sich aber Petrus am besten für eine Rolle, die den Grundstein einer Definition der Kirche als Raum der Gerechtigkeit darstellen konnte (Mt 16,13-20). ${ }^{9}$

Die Dimension dieses Dialogs als Gründungsereignis verlangt nun eine klare Definition beider Gesprächspartner: Das Christusbekenntnis des Petrus bleibt einerseits formal, so lange die Bedeutung und die anthropologisch-theologische Relevanz des Begriffes "Christus" nicht präzisiert wird. Dazu dient in der matthäischen Argumentation die Offenbarung der Notwendigkeit des Weges nach Jerusalem, der

\footnotetext{
9 Eine genaue Argumentation mit kritischem Rückblick auf die - späte - Rezeptionsgeschichte findet sich in Rinaldo Fabris, Matteo, Seconda edizione riveduta e aggiornata, Commenti biblici, Rom 1996, 365-374.
} 
Leiden, des gewaltsamen Todes und der Auferstehung (Mt 16,21), auf welche noch zweimal hingewiesen werden wird (Mt 17,22-23 und Mt 20,17-19). Aber der neue Name des Petrus und die Metaphorik des Steines wären andererseits genauso missverständlich, wenn die zweite Episode des Dialogs sie nicht verdeutlichen würden. Grundlegend für die mögliche Definition der Ekklesia als Raum der Gerechtigkeit und der Wahrhaftigkeit, gegen welche der Tod keine Macht haben wird, ist die Offenbarung der Ambivalenz der Person des Petrus, der sich sowohl als bekennender Jünger als auch als Verführer zeigen kann. In dem Ruf zur Nachfolge wird diese Ambivalenz in ihrer Gleichzeitigkeit transparent (Mt 16,22-23):

(22) Und, ihn beiseite genommen habend, fing Petrus an, ihn zu bedrohen: Auf dass du behütet seist, Herr! Das soll dir keinesfalls geschehen!

(23) Sich umgewandt habend sagte er zu Petrus:

Fort mit dir, Satan, hinter mich!

Für mich bist du ein Stolperstein, weil du nicht das Göttliche denkst, sondern das Menschliche.

Der programmatische Charakter des Dialogs und der notwendigen Veränderungen, die die Definitionen des Christus und seines Jüngers ankündigen, zeigen die drei Erläuterungen, die sich daran anschliessen.

2. Das Markusevangelium hatte den Dialog zwischen Petrus und Jesus (Mk 8,31-33) mit einer Kette von Aphorismen kommentiert, die Jesus an die Öffentlichkeit richtet, die er samt seinen Schülern zusammengerufen hat (Mk 8,34-38). Damit baut Markus eine Sequenz auf, die sich mit der Wahrheit der menschlichen Existenz und desVerhältnisses des Subjektes zu sich selbst befasst:Die Wahrheit der menschlichen Existenz besteht in der Grundhaltung des Vertrauens (vgl. Mk 11,22!), die sich in der Befreiung von der Sucht äussert, sich selbst retten zu wollen, und in der Freiheit, sein inneres und äusseres Leben als Geschenk wahrzunehmen. Matthäus hat diese Kette fast ohne Veränderungen übernommen, aber den Adressatenkreis der Sprüche Jesu auf die Jünger reduziert (Mt 16,24-26):

(24) Dann sagte Jesus seinen Jüngern:

Wenn jemand hinter mich kommen will,

verleugne er sich

und nehme sein Kreuz

und folge mir! 
(25) Denn

wer seine Seele retten will, wird sie verlieren;

wer seine Seele meinetwegen verlieren wird, wird sie finden.

(26) Denn

was wird es dem Menschen nützen, wenn er die ganze Welt gewinnt, aber seine Seele beschädigt?

Oder was wird ein Mensch als Tauschmittel seiner Seele geben?

Damit hat Matthäus auch die Bedeutung der Lehre verschoben: Sie definiert durch ein neues praktisches Selbstverständnis die universale Befindlichkeit der Jünger aller Zeiten, die in die im paradigmatischen Dialog zwischen Jesus und Petrus initiierten Ich-Du-Beziehung eintreten werden (Mt 16,24-28).

3. Unmittelbar angeschlossen an die Kette der Aphorismen hatte Matthäus im Markusevangelium bereits die sogenannte Verklärungsgeschichte gefunden (Mk 9,1-13 und Mt 17,1-14). Die Szene, in der sich symbolische Motive der alttestamentlichen Theophanie konzentrieren, bildet den Rahmen für die Erklärung der Stimme, die aus der Wolke heraus Jesus als ihren geliebten Sohn vorstellt und die vorhandenen Jünger auffordert, ihm zuzuhören (Mt 17,5):

Dies ist mein geliebter Sohn, an dem ich Wohlgefallen habe.

Hört auf ihn!

Wenn es um Gott geht, bleiben die beiden ersten Evangelien ausgesprochen diskret. Matthäus kennt zwar Engel, die im Traum oder vor dem leeren Grab erscheinen. Sonst folgt er aber dem Markusevangelium, in dem Gott nur zweimal als Subjekt auftritt, und zwar als Stimme, die Jesus als seinen Sohn bezeichnet und autorisiert (Mk 1,11 und Mt 3,17; Mk 9,7 und Mt 17,5). Gott wird also dadurch gekennzeichnet, dass er die Präsenz einer Transzendenz personifiziert, die die Wirklichkeit jenseits ihrer selbst begründet und die Worte Jesu in der Art als Wahrheit beglaubigt, dass er die Jünger - und die Leser - vor ihre eigeneVerantwortung stellt. Es fällt auf, dass die drei Jünger, die Jesus auf den Berg begleitet haben, vor Ostern nicht erzählen sollen, was sie gesehen haben. DieVision bildet aber nur den Rahmen dessen, was ihnen die Stimme gesagt hat und was sie gehört haben. Sowohl für Markus als auch für Matthäus steht 
der Inhalt im Zentrum der Erzählung und darf jederzeit weitervermittelt werden: Hört auf ihn!

4. Die vierte gründende Dimension der neuen Gemeinschaft der Ekklesia besteht im von dem geliebten Sohn zugetrauten, vermittelten und herausgeforderten Vertrauen. Noch einmal folgt Matthäus dem Aufbau des Markusevangeliums. Auch bleibt er beim Thema, das im Zentrum der markinischen Erzählung stand (Mk 9,14-29). Markus ging es um die existentielle Haltung des Vertrauens, die Undenkbares möglich macht (Mk 9,23-24):

\section{(23) Jesus sagte zu ihm:}

Was das "wenn du kannst « betrifft, alles ist dem möglich, der vertraut.

(24) Sofort aufschreiend, der Vater des Kindes sagte: Ich glaube, hilf meinem Unvertrauen!

Matthäus streicht den bei Markus entscheidenden Dialog zwischen Jesus und dem Vater, um die gesamte Dramaturgie in eine Szene des Gemeindelebens umzugestalten (kýrie eléēson), die dem Kleinglauben der Jünger das Vertrauen des Vaters, der den geliebten Sohn um Hilfe ruft, gegenüberstellt.

\section{Erster Schluss}

Als ersten Ertrag stelle ich fest, dass Matthäus den Stoff, den er in der Komposition des markinischen Evangeliums findet, genauso kreativ wiederverwertet und dass er aus der ersten Episode des Vertrauensweges Jesu nach Jerusalem (Mk 8,27-9,29) eine Geschichte der Gründung der Ekklesia schreibt (Mt 16,13-17,20). Damit legt er die Basis für die Selbstdefinition einer christlichen Kirche, die sich weder als eine dogmatisch festgelegte Grösse noch als eine heilige Einrichtung noch als eine religiöse Institution versteht; sondern als die offene Gemeinschaft einzelner Menschen, die vom Grundstein eines gründenden Dialogs lernen, aus Vertrauen und aus gegenseitiger Anerkennung in der Zeit, die ihnen gegeben ist (Wer seine Seele retten will $[\ldots])$ zu leben. ${ }^{10}$

Diese gedankliche, anthropologisch-theologische Grundlage bereitet den Leser für eine zweite Sequenz vor, die den Weg der markinischen Vorlage öfter verlassen wird, um eine originelle Ethik

${ }^{10}$ Vgl. François Vouga/Henri Hofer et André Jantet, Dieu sans religion. Les origines laïques du christianisme, Essais bibliques 51, Genf 2016. 
der Kommunikation auszuformulieren, die das matthäische Gemeindeverständnis kennzeichnet.

\section{Zweite Sequenz: Matthäus 17,22-18,35}

\section{Der Aufbau der Ekklesia als Gemeinschaft der Freiheit und der Wahrhaftigkeit}

1. Diese zweite Sequenz beginnt wieder mit einem Dialog zwischen Petrus und Jesus. Unmittelbar nach der zweiten Ankündigung der Auslieferung, des gewaltsamen Todes und der Auferstehung des Menschensohnes (Mt 17,22-23) wird die Grundlage der matthäischen Ethik der Kommunikation durch eine präzise Definition der evangelischen Freiheit gelegt (Mt 17,24-27). Die an Petrus - und nicht an Jesus - gerichtete Frage, ob sein Lehrer die Tempelsteuer zahlt oder nicht, stellt die Frage des Selbstverständnisses der in Mt 16,13-17,20 gegründeten Ekklesia. Die Antwort Jesu besteht aus einem Spruch und einem Kommentar, die zusammen genommen die Grundregel formulieren, die in der Gemeinschaft der Königsherrschaft der Himmel gilt (Mt 17,26-27):

Die Söhne sind frei, aber ihre Freiheit wird durch die Aufmerksamkeit begleitet, keinen Anstoss zu erregen.

In die Sprache der Paulusbriefe übersetzt:

Alles ist erlaubt, aber nicht alles baut auf (1 Kor 10,23, vgl. 6,12).

2. Dieser thetischen Antwort schliesst Matthäus die Frage der Jünger nach dem Grössten in der Königsherrschaft der Himmel (Mt 18,15 ) und die Warnungen Jesu vor der Gefahr an, für die Kleinen, die vertrauen, zum Anstoss zu werden (Mt 18,6-10). Ferner folgen zwei Durchführungen des Themas der Freiheit:

- Wer ist der Grösste in der Gemeinschaft der Freiheit der Königsherrschaft der Himmel? Die Gegenwart des Kindes eröffnet den Weg für eine pragmatische und paradoxe Definition: Die Haltung der Freiheit der Königsherrschaft besteht in der Aufnahmebereitschaft der Kleineren. 
- Aus den paradoxen Definitionen der Freiheit durch die Grenze der Aufmerksamkeit auf die Anderen (Mt 17,26-27) und der wahren Grösse in der Gemeinschaft der Ekklesia als die Freiheit, die Kleinen aufzunehmen (Mt 18,1-5), folgt notwendigerweise, dass derjenige oder diejenige, der oder die bei Kleineren Anstoss erregt, sich selbst von der Freiheit der Söhne und Töchter ausgeschlossen hat oder ausschliesst.

3. Aus seiner Definition der Freiheit der Königsherrschaft der Himmel leitet Matthäus drei notwendige und ausreichende praktische Regeln für den Aufbau der Ekklesia als Gemeinschaft der Freiheit und der Wahrhaftigkeit $\mathrm{ab}$.

Erste Regel: Die aktive Aufmerksamkeit auf die Kleinen und die Verantwortung, diejenigen, die sich verlieren, zurückzuholen. Genauso, wie Matthäus die Stoffe, die er vom Markusevangelium übernommen hat, in die Logik seiner eigenen Argumentation verarbeitet und einbaut, so aktualisiert er jetzt das Gleichnis des verlorenen Schafes als erstes Element einer offensiven Ethik der Kommunikation (Mt 18,10-14; Lk 15,3-7):

(10) Seht $z u$, dass ihr nicht anfangt, einen dieser Kleinen zu verachten!

Denn ich sage euch:

Ihre Engel schauen allezeit das Angesicht meines Vaters in den Himmeln.

(14) So ist es nicht der Wille vor eurem Vater in den Himmeln, dass eins dieser Kleinen anfange, verloren zu gehen.

Die matthäische Ekklesia definiert sich zunächst dadurch als Gemeinschaft der Freiheit und der Wahrhaftigkeit, dass sich die gegenseitige Anerkennung nicht auf ein passives non nocebo reduzieren kann, sondern dass die im Anschluss an den paradigmatischen Dialog zwischen Jesus und Petrus aufgebauten Ich-Du-Beziehungen eine aktive, verantwortliche, offensive - im Sinne von einer nicht bloss reaktiven oder defensiven - Haltung und Verhaltensweise der Jünger untereinander und füreinander voraussetzt.

Zweite Regel: Die brüderliche Disziplin der aufrichtigen und respektvollen Kritik (Mt 18,15-20):

(15) Wenn dein Bruder anfängt, zu sündigen,

geh hin,

überzeuge ihn zwischen dir und ihm allein! 


\begin{tabular}{|c|c|c|}
\hline \multicolumn{3}{|l|}{$\begin{array}{l}\text { - Wenn er auf dich hört, } \\
\text { hast du deinen Bruder } \\
\text { gewonnen. }\end{array}$} \\
\hline & $\begin{array}{l}\text { - (16) Wenn er nicht hört, } \\
\text { Nimm mit dir einen oder } \\
\text { zwei, damit alles durch } \\
\text { zweier oder dreier Zeu- } \\
\text { gen Mund geregelt sei. }\end{array}$ & \\
\hline & & $\begin{array}{l}\text { - (17) Wenn er an ihnen } \\
\text { vorbei hört, } \\
\text { sprich zur Ekklesia! } \\
\text { - Und wenn er an der } \\
\text { Ekklesia vorbei hört, } \\
\text { sei er für dich wie der } \\
\text { Heide und der Zöllner! }\end{array}$ \\
\hline
\end{tabular}

(18) Amen, ich sage euch:

alles, was ihr auf Erden binden werdet, wird im Himmel gebunden sein, und alles, was ihr auf Erden lösen werdet, wird im Himmel gelöst sein.

(19) Weiter sage ich euch:

wenn zwei unter euch auf Erden übereinstimmen

über alles, was sie bitten werden,

dann wird es ihnen von meinem Vater, der in den Himmeln ist, geschehen.

(20) Denn

da wo zwei oder drei in meinem Namen versammelt sind, da bin ich mitten unter ihnen.

Diese zweite Regel schliesst sich unmittelbar an die erste an. Sie ergänzt sie durch eine Strategie der konstruktiven Kritik. Worin die »Sünde« bestehen kann, spielt keine Rolle. Sie kann alles sein, was in "deinen« Augen als problematisch erscheinen kann.

- Entscheidend für die matthäische Ethik der Kommunikation ist zunächst, dass die Kritik nicht über Dritte kommuniziert wird, sondern direkt gegenüber den Betreffenden. ${ }^{11}$

${ }^{11}$ Konsequent sind die sogenannten Weherufe Jesu (Mt 23,13-39) keine "Weherufe über Schriftgelehrte und Pharisäer", als ob die Schriftgelehrten und Pharisäer die Objekte einer Kritik in der dritten Person wären und als ob Matthäus seine Leser einladen würde, mit ihm zusammen über sie zu lästern. Matthäus formuliert vielmehr seine Kritik unter der Form von Invektiven zu den Menschen, die er direkt in 
- Entscheidend ist dann, dass Matthäus wiederum und konsequent $\mathrm{zu}$ einer aktiven Verantwortung und $z u$ einem offensiven Verhalten auffordert: Das erste Imperativ besteht in der Aufgabe, zu dem Bruder hinzugehen, und das zweite in dem Auftrag, bei ihm Überzeugungsarbeit zu leisten.

- Entscheidend ist drittens die Abfolge der verschiedenen Gesprächskreise, die sich erst dann ergänzen, falls es sich als notwendig erweist. Die genaue Beschreibung der verschiedenen Stufen lässt keinen Zweifel über das Ziel der sorgfältig durchdachten Kommunikationsstrategie zu. Der Rahmen der IchDu-Beziehung unter vier Augen wird erst dann erweitert, wenn sich der Dialog als unmöglich erweist; die anderen Mitglieder der Gemeinde sollen erst dann damit befasst werden, wenn die Vermittlung zweier oder dreier Zeugen gescheitert ist. Und am Ende, falls keine Verständigung möglich scheint, soll er von »dir« - nicht von der ganzen Gemeinschaft oder von der Ekklesia wie der Heide und der Zöllner betrachtet werden, dass heisst wie Menschen, über welche der himmlische Vater seine Sonne aufgehen und regnen lässt, und die da sind, um geliebt und in die Königsherrschaft der Himmel eingeladen und aufgenommen zu werden. Die matthäische Strategie der konstruktiven, aufrichtigen und respektvollen Kritik in der Gemeinde hat folglich ihren Sinn in der praktischen Ausübung der Nächstenliebe. Ihr Ziel besteht weder in der vermeintlichen Rettung einer institutionellen Ordnung der Ekklesia noch im vergeblichen Versuch, das Vollkommenheitsideal einer heiligen, sündlosen Gemeinschaft zu verwirklichen, sondern in der Wahrnehmung derVerantwortung jedes einzelnen Mitglieds der Gemeinde, dass keiner der kleinsten oder grössten Brüder anfange, verloren zu gehen.

- Entscheidend ist viertens die symbolische Bedeutung der persönlichen Haltungen und der praktischen Verhaltensweisen in der Gemeinde. Dem Matthäusevangelium ist nämlich bewusst, dass mit der Verachtung oder mit der Aufnahme der Kleinen, mit der vermeintlichen Freiheit, die man sich nimmt, Anstoss zu erregen, oder umgekehrt mit der verantwortungsbewussten Aufmerksamkeit auf die Kleinen, die anfangen, verloren zu gehen, nicht nur die immanente, soziale Komposition der "auf Erden" real existierenden Gemeinden steht, sondern auch das Vertrauen in eine Transzendenz »in den Himmeln«, die die bedingungs-

der zweiten Person - als Gesprächspartner - anspricht, um sie vor der Situation der Heuchelei, in der sie sich nach dem matthäischen Jesus befinden und die er ihnen offenbart, zu warnen. 
lose Anerkennung jedes einzelnen Menschen als freies und verantwortliches Subjekt jenseits jeder immanent feststellbaren Eigenschaften begründet.

\begin{tabular}{|c|c|}
\hline $\begin{array}{l}\text { Die Metapher des Schlüssels: In } \\
\text { der Internationalität des Petrus } \\
\text { findet der Universalismus der Ekk- } \\
\text { lesia seine Entsprechung und seinen } \\
\text { Massstab. (Mt 16,19) }\end{array}$ & $\begin{array}{l}\text { Die Verantwortung der Jünger in } \\
\text { der Ekklesia, die gegründet wurde, } \\
\text { um offener Raum der Freiheit, der } \\
\text { gegenseitigen Anerkennung und } \\
\text { der Wahrhaftigkeit auf Erden zu } \\
\text { sein. (Mt 18,18) }\end{array}$ \\
\hline $\begin{array}{l}\text { (19) Ich werde dir die Schlüssel } \\
\text { der Königsherrschaft der Himmel geben, } \\
\text { - und was du auf Erden binden } \\
\text { wirst, } \\
\text { wird in den Himmeln gebun- } \\
\text { den sein } \\
\text { - und was du auf Erden lösen } \\
\text { wirst, } \\
\text { wird in den Himmeln gelöst } \\
\text { sein. }\end{array}$ & $\begin{array}{l}\text { (18) Amen, ich sage euch: } \\
\text { - soviel ihr auf Erden binden } \\
\text { werdet, } \\
\text { wird im Himmel gebunden sein, } \\
\text { - und soviel ihr auf Erden lösen } \\
\text { werdet, } \\
\text { wird im Himmel gelöst sein. }\end{array}$ \\
\hline
\end{tabular}

Dritte Regel: Die paradoxe Neudefinition der Vergebung schliesst sich wiederum unmittelbar an die beiden ersten Regeln an. Eingeleitet wird sie durch den Dialog zwischen Petrus und Jesus (Mt 18,21-22):

(21) Dann sagte ihm Petrus zu ihm hervortretend:

Herr,

wie oft wird mein Bruder gegen mich sündigen,

und ich werde ihm vergeben? Bis zu siebenmal?

(22) Jesus sagt ihm:

Ich sage Dir nicht bis zu siebenmal, sondern bis zu siebzigmal siebenmal.

Dem Leser wird sofort klar, dass die arithmetische Ausdehnung einen Systemwechsel bedeutet, und dass das Vergebungsverständnis, das die matthäische Ethik der Kommunikation mit der Unendlichkeit der 77 oder 70 mal 7 vertritt, ${ }^{12}$ mit der Vergebung im eigentlichen Sinne nichts mehr zu tun hat. Die Vergebung wird nicht mehr

${ }^{12} \mathrm{Ob}$ Matthäus 70 plus 7 oder 70 mal 7 meint, ist sprachlich unentscheidbar, weist aber auf das Gleiche hin: Das matthäische Vergebungsverständnis verlässt jede Berechnungsmöglichkeit. Genaue Argumentation und ausführliche Diskussion in William 
als regulatives System gedacht, dessen Funktion es ist, durch einen Schuldenerlass dem Sünder die Möglichkeit zurück zu geben, eine so weit wie möglich befreite Existenz ohne Schulden - oder Sünden - zu fuihren, wie es das grosszügige Angebot des Petrus immer noch impliziert. Das unter der Autorität Jesu formulierte alternative Modell hebt jede Berechnung auf, um einem Geist der Umsonstheit Platz zu machen, in welchem jeder "Bruder« frei, aufmerksam und verantwortungsvoll mit der Freiheit seiner »Brüder« respektvoll umgehen kann.

Das Gleichnis vom unbarmherzigen Knecht, das Matthäus mit dem Dialog organisch verbindet (Mt 18,23-35), argumentiert ebenfalls mit masslosen Zahlen. Zum einen stellt zwar der König den unter der Autorität Jesu empfohlene Systemwechsel exemplarisch dar, indem er die Bitte des ersten Knechtes um eine Frist durch einen radikalen Schuldenerlass beantwortet. Aber noch fast unvorstellbarer erscheint die Höhe der Schuld, um welche es geht. Damit führt uns Matthäus zu einem für ihn wichtigen Begriff, der schon in seiner Fassung des Vaterunsers eine zentrale Rolle ausdrücklich spielt, der seinem Verständnis der gegenseitigen Anerkennung eine präzise Formulierung gibt und eine klare anthropologische Grundlage für seine Vorstellung der Ekklesia als Gemeinschaft der Freiheit und der Wahrhaftigkeit bringt: der Begriff solidarischer Verbindungen durch vergebene Schulden ${ }^{13}$ : Er setzt sich zusammen aus dem Bekenntnis der Fehlbarkeit und aus Verbindlichkeit, aus gegenseitiger Freiheit und aus Dankbarkeit, aus dem Bewusstsein der eigenen Schwachheit und aus einer aufmerksamen Verantwortung für den »kleinen Bruder».

\section{Zweiter Schluss}

Als zweiten Ertrag stelle ich die Hypothese auf, dass der deutsche Begriff der Wahrhaftigkeit zwar kein direktes Äquivalent im Matthäusevangelium - und, wie ich denke, im neutestamentlichen Corpus insgesamt - findet, aber dass die Reflexion des Matthäus über die Kirche als Ekklesia des gekreuzigten Herrn und als Gemeinschaft des Vertrauens und der Freiheit eine Ethik der Kommunikation entwickelt, die die notwendigen und ausreichenden Bedingungen erfüllt, um die christliche Gemeinde als Raum des zwischenpersön-

David Davies/Dale C. Allison, The Gospel According to Saint Matthew II: Commentary on Matthew VIII-XVIII, ICC, Edinburgh 1991, 793.

${ }_{13}$ Bernard Piettre/François Vouga, La dette. Enquête philosophique, théologique et biblique sur un mécanisme paradoxal, Essais bibliques 49, Genf 2015, 13-34. 
lichen Respekts, der offenen und kritischen Suche nach Wahrheit und folglich der Wahrhaftigkeit definiert.

- François Vouga ist emeritierter Professor für Neues Testament an der Kirchlichen Hochschule Wuppertal/Bethel. Neben der Einheit und Vielfalt der neutestamentlichen Theologie sowie Paulus, zählen die nicht religiöse Ekklesiologie des Matthäusevangeliums und die theologische Anthropologie des Markusevangeliums zu seinen Forschungsschwerpunkten. Besonderes Interesse hat er zudem an interdisziplinären Kooperationen mit der Medizin, der Kunst und der Musik. 